\title{
Variaciones espacio-temporales de la captura por unidad de esfuerzo en la pesquería artesanal costera del pargo rayado Lutjanus synagris, en el Caribe colombiano y su relación con variables ambientales
}

\author{
Spatio-temporal variations of the catch per unit effort in the coastal small-scale fishery of snapper \\ Lutjanus synagris, of the Colombian Caribbean and their relationship with environmental variables \\ Diana P. Párraga ${ }^{1,2}$, Luis A. Cubillos ${ }^{3}$ y Marco A. Correa-Ramirez ${ }^{4}$ \\ ${ }^{1}$ Departamento de Evaluación de Pesquerías, Instituto de Fomento Pesquero, Blanco Encalada 839, Valparaíso, Chile \\ ${ }^{2}$ Programa de Magíster en Ciencias con Mención en Pesquerías, Universidad de Concepción, Concepción, Chile \\ ${ }^{3}$ Departamento de Oceanografía, Facultad de Ciencias Naturales y Oceanográficas, \\ Universidad de Concepción, Concepción, Chile \\ ${ }^{4}$ Departamento de Geofísica,Universidad de Concepción, Concepción, Chile \\ diana.parraga@ifop.cl
}

\begin{abstract}
The coastal small-scale fishery in the Colombian Caribbean is characterized by the joint exploitation of demersal and pelagic species, which are caught using a variety of fishing gears. The snapper Lutjanus synagris, is one of the commercially important demersal fish species which is distributed throughout the Caribbean coast. We studied the spatio-temporal changes in the catch per unit effort (CPUE) of snapper, using data coming from the logbooks of the smallscale longline fishery. These data were obtained from hauls carried out in the Magdalena Department (73⒊' W-750'W) between 1994 and 2001. The CPUE was standardized using Generalized Linear Models (GLM) considering year, month and zone of landings as factors. We also studied the relationship between CPUE with sea surface temperature (SST) and the runoff of the Magdalena River. The results showed important interannual changes in the CPUE. Although changes in CPUE are usually associated with changes in abundance, the standardized CPUE could obey mostly to changes in fishing power. Coefficients associated with the monthly factor showed the higher values CPUE during the rainy season (AugustDecember) and between December-March, related with migrations of the demersal snapper ("bajanza"). No significant relationship between the SST and the CPUE was found. However, a significant and positive relationship was found between the CPUE and the discharge of the Magdalena River with a lag between one to three months. This relationship could be associated with an increment in the productivity due to the input of nutrients through of the river discharge. This process may affect positively the prey availability for snapper.
\end{abstract}

Key words: Standardization, GLM, temperature, runoff, multispecies fishery
Resumen.- La pesquería artesanal costera del Caribe colombiano se caracteriza por la explotación conjunta de especies demersales y pelágicas, utilizando gran variedad de artes de pesca. Entre los recursos de importancia económica destaca el pargo rayado Lutjanus synagris, especie demersal distribuida en todo el Caribe. Utilizando datos de la flota palangrera artesanal que operó en el Departamento del

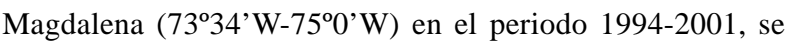
estudió la variabilidad espacial y temporal en la captura por unidad de esfuerzo (CPUE) del pargo rayado. La CPUE se estandarizó mediante Modelos Lineales Generalizados (MLG) considerando como factores el año, mes y zona de desembarque. Además se estudió la relación entre la CPUE con la temperatura superficial del mar (TSM) y el caudal del río Magdalena. Los resultados mostraron variaciones interanuales importantes en la CPUE. Aunque podrían estar asociadas a la abundancia del stock, dichas variaciones se explicarían por cambios en el poder de pesca de las embarcaciones. Los coeficientes mensuales indicaron una CPUE alta durante la estación lluviosa (agostodiciembre) y entre diciembre-marzo relacionado a un proceso migratorio de la especie (bajanza). En este trabajo no se encontró una relación significativa entre la CPUE y la TSM. No obstante, la CPUE respondió positivamente a la influencia del caudal del río Magdalena con uno a tres meses de retraso. Esta relación podría ser consecuencia de un incremento en la productividad por aporte de nutrientes de la descarga del río. Este proceso puede afectar positivamente en la disponibilidad de presas para el pargo rayado.

Palabras clave: Estandarización, MLG, temperatura, descarga, pesquería multiespecífica 


\section{Introducción}

En la costa del Caribe colombiano se desarrollan tres tipos de pesquerías: (1) La pesquería artesanal costera (PAC), con embarcaciones pequeñas de fibra de vidrio y/o madera; (2) La pesquería artesanal avanzada, con lanchas de mayor autonomía que ejercen una pesca a mayores profundidades; y (3) La pesquería industrial, representada por los barcos de arrastre demersal dedicados a la explotación de camarón (Penaeus spp.). Este estudio se enfocó en la pesquería artesanal costera (PAC) del Departamento del Magdalena que opera desde la desembocadura del río Palomino (733'' W) hasta la desembocadura del río Magdalena $\left(75^{\circ} 0^{\prime} \mathrm{W}\right)$, cuya extensión es determinada por la distancia a los centros de acopio y las condiciones de navegación, restringiéndose hasta la isobata de los 200 m (Fig. 1; Gómez-Canchong et al. 2004, Cuello et al. 2005).
La PAC ha desembarcado cerca del 56\% del total de las capturas del Caribe colombiano y ha generado alrededor de 4000 empleos directos e indirectos en la región (Barros \& Manjarrés 2004, Correa \& Manjarrés 2004). Dentro de los desembarques se registra una gran diversidad de especies ( $c a$. 200 especies) capturadas con múltiples artes de pesca (líneas de mano, nasas, palangres, chinchorros y redes de enmalle), aunque solo 10 grupos representan más del 70\% del total de la captura (Duarte \& García 2002). Dentro de este grupo está el pargo rayado, Lutjanus synagris Linnaeus, 1758 considerado de gran valor comercial y ampliamente distribuido en el mar Caribe de Colombia (Manjarrés 2004b). Habita en fondos rocosos como arrecifes coralinos y fondos blandos y es capturado principalmente con palangre entre los 14 y 80 m de profundidad (García et al. 1998, Manjarrés 2004b).

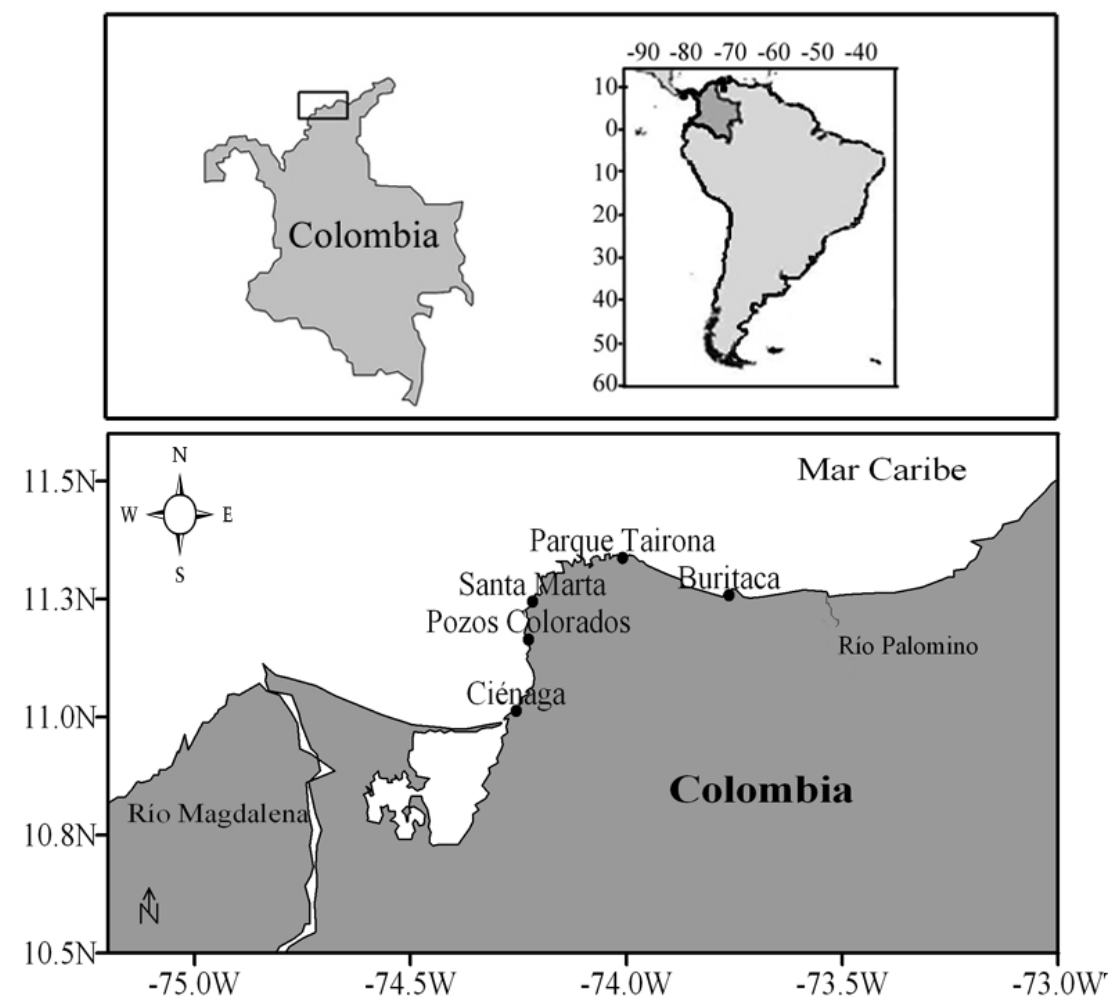

Figura 1

Área de influencia de la pesquería artesanal del Departamento del Magdalena noreste del Caribe colombiano (los puntos representan los puertos de desembarque)

Influence area of the small-scale fishery, Department of Magdalena, north east of the Colombian Caribbean (points represent landing zone) 
Se estimó que las capturas de pargo rayado en el periodo de 1994-2001 fueron de 298 t, siendo el mayor valor desembarcado en 1998 con 71 t. Esta especie ha presentado variaciones estacionales en sus capturas y captura por unidad de esfuerzo, con alta abundancia y frecuencia en las capturas en época lluviosa (agosto a diciembre) y no solo relacionado con su ciclo vital, sino también por la influencia del ambiente (Barros \& Manjarrés 2004). La PAC en el Caribe colombiano se desarrolla principalmente en la región costera, la cual se encuentra directamente influenciada por el sistema de surgencia de la Guajira y el aporte continental río Magdalena (Andrade \& Barton 2005). Estas variables provocan una marcada estacionalidad y determinan la productividad del ecosistema (Blanco 1988), por lo cual es de fundamental importancia conocer la influencia ambiental sobre la distribución, abundancia y posibles migraciones en el análisis de poblaciones de peces explotados (Beamish 1995, Sinclair \& Frank 1995).

Dada la importancia del pargo rayado en la PAC del Departamento del Magdalena, es indispensable un adecuado monitoreo de su estado de explotación. En este estudio, se analizaron datos de desembarque y esfuerzo de pesca que cubren el periodo 1994-2001 con el objeto de determinar las principales fuentes de variabilidad en la CPUE del pargo rayado. Asimismo, con el objeto de establecer la influencia del ambiente sobre la disponibilidad de este recurso se exploran relaciones con algunas variables ambientales características del Caribe colombiano.

\section{Material y métodos}

El área de estudio está referida a la actividad de la PAC realizada en el Departamento del Magdalena en el Caribe colombiano (Fig. 1). Los datos fueron obtenidos del Sistema de Procesamiento de Información de Captura y Esfuerzo Pesquero (PICEP), descrito por Manjarrés (2004a). Se utilizaron registros diarios de desembarques para la especie (sitio de desembarque, profundidad, artes de pesca y posición geográfica del área de pesca) y datos de esfuerzo de pesca, estrato geográfico y tipo de arte de pesca, desde 1994 hasta junio de 2001, exceptuando el año 1999. Con esta información, se analizaron las estadísticas del pargo rayado capturado con palangre de fondo, que representa los principales desembarques de esta especie. En la PAC del Caribe colombiano el descarte es inusual, de tal manera que los desembarques son prácticamente equivalentes a las capturas (Manjarrés 2004a). La CPUE, fue calculada dividiendo el peso total de la captura por especie $(\mathrm{kg})$ por el esfuerzo de pesca, este último medido en número de horas de faena de pesca (hf). El área de estudio fue estratificada en cinco zonas geográficas, de acuerdo a las características comunes de la actividad pesquera y del hábitat, referidos al sitio de desembarque más representativo en cada zona: Ciénaga $\left(74,33^{\circ} \mathrm{W}-74,25^{\circ} \mathrm{W}\right.$, Zona 1$)$, Pozos colorados $\left(74,23^{\circ} \mathrm{W}\right.$ $74,22^{\circ} \mathrm{W}$, Zona 2$)$, Santa Marta $\left(74,22^{\circ} \mathrm{W}-74,21^{\circ} \mathrm{W}\right.$, Zona $3)$, Parque Tayrona $\left(74,19^{\circ} \mathrm{W}-74,00^{\circ} \mathrm{W}\right.$, Zona 4), y Buritaca $\left(73,86^{\circ} \mathrm{W}-73,57^{\circ} \mathrm{W}\right.$, Zona 5). Esta clasificación se realizó sobre la base de los registros totales de pesca, más en el período evaluado no se obtuvo registros de captura del pargo rayado en la Zona 3.

Se utilizó un Modelo Lineal Generalizado, implementado en el programa estadístico R (Ihaka \& Gentleman 1996), para evaluar la variación de la CPUE ( $\mathrm{kg} \mathrm{hf}^{-1}$ ) en función de factores como la zona de desembarque, año y mes. Para el ajuste se utilizó la distribución de probabilidad Gaussiana y el enlace identidad que permitió describir adecuadamente la distribución del error de la variable respuesta. Esta selección se basó en la relación media-varianza de la CPUE mensuales, donde la varianza debe ser proporcional al cuadrado de la media (McCullagh \& Nelder 1989).

El modelo implementado se expresó de la forma $g(E(Y))=\eta$, donde $\eta=\beta_{0}+\alpha_{y}+\beta_{j}+\gamma_{\kappa}+\varepsilon_{y j k}$, Y es la variable respuesta y corresponde al logaritmo de la CPUE nominal, g(•) es la función de enlace, $\eta$ es el predictor lineal, $\beta_{0}$ es la media total del $\log _{\mathrm{e}}(\mathrm{Y}), \alpha_{y}+\beta_{j}+\gamma_{\kappa}$ son los efectos del año $(y)$, mes $(j)$ y zona de desembarque $(k)$, y $\varepsilon$ es el término de error (McCullagh \& Nelder 1989). El año se consideró como un factor ya que el objetivo primario de la estandarización es detectar tendencias interanuales asociados con la abundancia. Con el objetivo de establecer su significancia, las variables que ingresaron al modelo fueron evaluadas con un ANOVA a partir de del estadístico F (Allen \& Punsly 1984). La devianza fue utilizada para determinar el porcentaje de explicación de cada factor sobre la variabilidad de la CPUE, la que es definida en los MLG como la diferencia entre la log-verosimilitud de un modelo saturado y la log-verosimilitud del modelo ajustado (similar al coeficiente de determinación $\left(\mathrm{r}^{2}\right)$ de una suma de cuadrados común), siendo $\mathrm{D}(y, \theta)=2 \log$

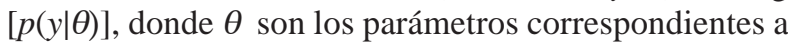
la distribución de la variable y (Zuur et al. 2007).

Las variables oceanográficas analizadas fueron temperatura superficial del mar $\left({ }^{\circ} \mathrm{C}\right)$ y el caudal del río Magdalena $\left(\mathrm{m}^{3} \mathrm{~s}^{-1}\right)$. La información satelital de temperatura superficial del mar, correspondió al producto diario del AVHRR Pathfinder versión 5.0, con 4 km de resolución, distribuido por la $\mathrm{NOAA}^{1}$, limitándose al

\footnotetext{
${ }^{1}$ National Oceanic and Atmospheric Administration (NOAA) $<$ http://www.noaa.gov/>
} 
Vol. 45, Nº1, 2010

periodo de 1994-2001. La información fue interpolada previamente con una aplicación Kriging en tres dimensiones (longitud-latitud-temperatura) para reducir el número de datos faltantes provocados por la cobertura nubosa. Posteriormente se construyeron matrices correspondientes a cada día, mes y año del periodo de estudio, mediante la interpolación lineal de los campos satelitales de temperatura. Los datos mensuales de caudal $\left(\mathrm{m}^{3} \mathrm{~s}^{-1}\right)$ del río Magdalena que desemboca en la zona de estudio, fueron obtenidos del Instituto de Hidrología Meteorología y Estudios Ambientales de Colombia (IDEAM), para el periodo 1994-2001.

Las series de tiempo de promedios mensuales de las variables ambientales, temperatura y caudal del río Magdalena fueron descompuestas para eliminar la tendencia global de los datos y la estacionalidad utilizando la función STL (Seasonal, Trend and irregular components using Loess) del paquete 'stats' disponible para el programa R (Ihaka \& Gentleman 1996). El procedimiento de descomposición STL esta basado en suavizadores Loess, lo que implica un algoritmo iterativo que progresivamente suaviza y mejora las estimaciones de la componente estacional y la tendencia, siendo resistente a observaciones extremas (Cleveland et al. 1990). Se utilizó una función de autocorrelación (FAC) para los residuos obtenidos en el proceso de descomposición con el objeto de evaluar el grado de dependencia de los datos a un retraso máximo de 12 meses. En este caso, si la secuencia se distribuye independiente e idénticamente distribuida (IID $\approx \mathrm{N}[0$, $\left.\sigma^{2}\right]$ ), cerca del 95\% de los datos deberían caer entre los limites de $\pm 1,96 / \sqrt{ } \mathrm{n}$ (siendo 1,96 el cuantil de 0,975 en una distribución normal estándar). Si un coeficiente en particular es más grande en magnitud que $\pm 1,96 / \sqrt{ } \mathrm{n}$, se aseguraría en un 95\% que el coeficiente de autocorrelación no es cero (Brockwell \& Davis 2002). Posteriormente se utilizó una función de correlación cruzada (FCC) para detectar la dependencia entre los residuales de las series de tiempo ambientales y la CPUE (Venables \& Ripley 2002).

\section{Resultados}

Durante el periodo de 1994-2001 la PAC del Caribe colombiano registró una alta diversidad en los desembarques con más de 150 especies, aunque solo 10 de ellas representaron más del $70 \%$ de la captura total. La anchoveta del atlántico (Cetengraulis edentulus) representó el 29\% del desembarque total, esta especie es de escaso valor económico y es usada como alimento fresco en el cultivo de cocodrilos (Duarte \& García 2002). En relación con la especie objetivo de estudio, el pargo rayado (Lutjanus synagris) significó en este periodo un 2\%, equivalente a 298 t (Tabla 1) (Barros \& Manjarrés 2004). A pesar de su menor porcentaje en los
Tabla 1

\section{Especies de mayor captura en la pesquería artesanal} costera (PAC) del Caribe colombiano entre 1994-2001

Species mostly caught in the small-scale fishery of the Colombian Caribbean, between 1994 and 2001

\begin{tabular}{lrcc}
\hline Nombre científico & $\begin{array}{r}\text { Captura } \\
\text { total (t) }\end{array}$ & $\begin{array}{c}\text { Captura } \\
\text { total (\%) }\end{array}$ & $\begin{array}{c}\text { Captura } \\
\text { acumulada (\%) }\end{array}$ \\
\hline Cetengraulis edentulus & 3729,2 & 29 & 29 \\
Caranx crysos & 1002,9 & 8 & 37 \\
Auxis thazard & 1011,3 & 8 & 45 \\
Selar crumenophthalmus & 803,4 & 6 & 51 \\
Opisthonema oglinum & 824,6 & 6 & 57 \\
Sphyraena spp. & 697,4 & 5 & 63 \\
Caranx spp. & 468,5 & 4 & 66 \\
Scomberomorus spp. & 397,6 & 3 & 69 \\
Lutjanus analis & 359,7 & 3 & 72 \\
Lutjanus synagris & 298,9 & 2 & 74 \\
Otras spp. & 3297,2 & 26 & 100 \\
\hline
\end{tabular}

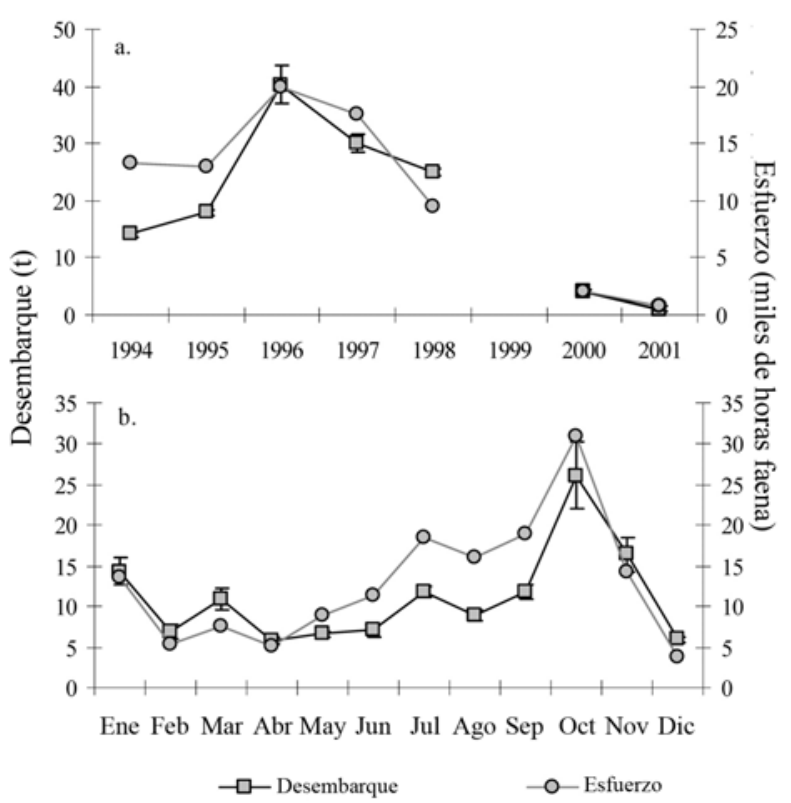

Figura 2

Serie histórica de desembarques (a) total anual y

(b) media mensual de pargo rayado (Lutjanus synagris), en el Departamento del Magdalena, Caribe colombiano entre 1994-2001

Historic landings of snapper (Lutjanus synagris),

(a) annual and (b) monthly mean, Department

of Magdalena, Colombian Caribbean during 1994-2001 
desembarques, esta especie es considerada de importancia económica y tiene el mayor número de registros de captura en la base histórica.

En la escala anual, los desembarques de pargo rayado capturado con palangre de fondo han sido menores a 50 t en el periodo de estudio. Se observó un aumento desde $14 \mathrm{t}$ en 1994 hasta $40 \mathrm{t}$ en 1996, para posteriormente presentar una disminución, alcanzando sólo 4 y 1 t en los últimos dos años. En el ciclo anual, el desembarque mensual y el esfuerzo de pesca se incrementan desde abril hasta octubre, alcanzando $26 \mathrm{t}$ asociado con un esfuerzo de pesca de 30 mil hf y decrecen hasta diciembre (Fig. 2)

En el diagrama de cajas se observó que el $\log _{\mathrm{e}}$ (CPUE) nominal mostró fluctuaciones anuales, con un incremento desde 1994 a 2000. En la escala estacional no se observaron diferencias significativas entre los meses, mostrando un ciclo unimodal con valores mayores en octubre y noviembre, y menores en mayo. En términos espaciales, en las zonas donde hubo actividad, se observaron diferencias significativas, con mayor $\log _{\mathrm{e}}$ (CPUE) en la zona 1 y 5 , y una menor $\log _{\mathrm{e}}$ (CPUE) en la zona 2 (Fig. 3).
La relación potencial varianza-media de los valores nominales mensuales de la CPUE, durante el periodo analizado, resultó no ser significativamente diferentes de la cuadrática $\left(P<0,05 ; r^{2}=0,83\right)$, sugiriendo que la distribución de probabilidad Gaussiana representó de mejor forma la variabilidad del $\log _{e}$ (CPUE).

Para la estandarización con MLG se utilizó un total de 987 registros entre mayo de 1994 y junio de 2001 de un total de 76 mil horas de faena aproximadamente. El modelo ajustado explicó el 31\% de la variabilidad total del $\log _{\mathrm{e}}$ (CPUE) del pargo rayado. La variable con mayor contribución fue el año con 14\%, seguido de la zona de desembarque con un 9\%, mientras que los meses sólo explicaron el 8\% (Tabla 2). El análisis de los residuos evidenció la presencia de valores de $\log _{\mathrm{e}}$ (CPUE) pequeños que no fueron explicados por el modelo de estandarización, indicando un pobre ajuste a partir de las variables incorporadas. Aunque no se detectaron tendencias, la presencia de algunos datos atípicos determinó que algunos residuos se alejaran de la distribución normal (Fig. 4).

Los resultados del modelo mostraron diferencias significativas de la variable respuesta, $\log _{\mathrm{e}}$ (CPUE), entre años siendo este factor año el de mayor explicación dentro
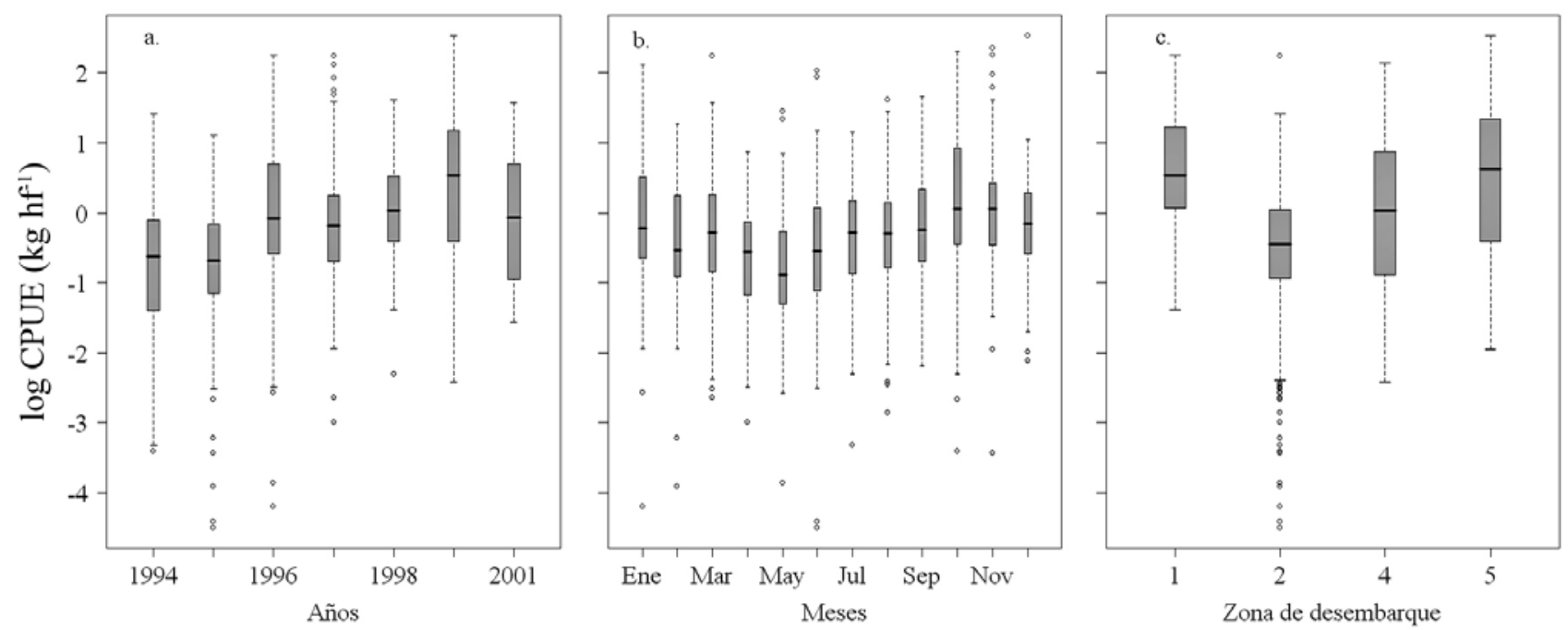

Figura 3

Diagrama de cajas (Box Plot) representando el logaritmo de la captura por unidad de esfuerzo nominal (kg hf $\left.{ }^{-1}\right)$ por año, mes y zona de desembarque, periodo 1994-2001. Zonas: 1=Ciénaga, 2 =Pozos Colorados, 4 =Parque Tayrona, 5=Buritaca

Boxplot of log transformed catch per unit of nominal effort $\left(\mathrm{kg} \mathrm{hf}^{-1}\right)$, for year, month and landing zone, 1994-2001 period. Zones: 1=Ciénaga, 2=Pozos Colorados, 4=Parque Tayrona, 5=Buritaca 


\section{Tabla 2}

Variables predictorias y porcentaje de la devianza explicada por el modelo ajustado para el pargo rayado (Lutjanus synagris)

Predicting variables and amount of deviance explained by the adjusted model for the snapper (Lutjanus synagris)

\begin{tabular}{lrrrrrrc}
\hline Variables & $\begin{array}{c}\text { Grados } \\
\text { de libertad }\end{array}$ & Devianza & $\begin{array}{c}\text { Grados } \\
\text { de libertad } \\
\text { residual }\end{array}$ & $\begin{array}{c}\text { Devianza } \\
\text { residual }\end{array}$ & $F$ & Valor $P$ & $\begin{array}{c}\text { Devianza } \\
\text { explicada (\%) }\end{array}$ \\
\hline & & & 986 & 910,48 & & & \\
Año & 6 & 125,34 & 980 & 785,14 & 32,214 & 0,001 & 14 \\
Mes & 11 & 73,01 & 969 & 712,13 & 10,235 & 0,001 & 8 \\
Sitio & 3 & 85,7 & 966 & 626,43 & 44,053 & 0,001 & 9 \\
Devianza del modelo & & 284,05 & & & & & 31 \\
Devianza residual & & 568,1 & & & & & 69 \\
\hline
\end{tabular}
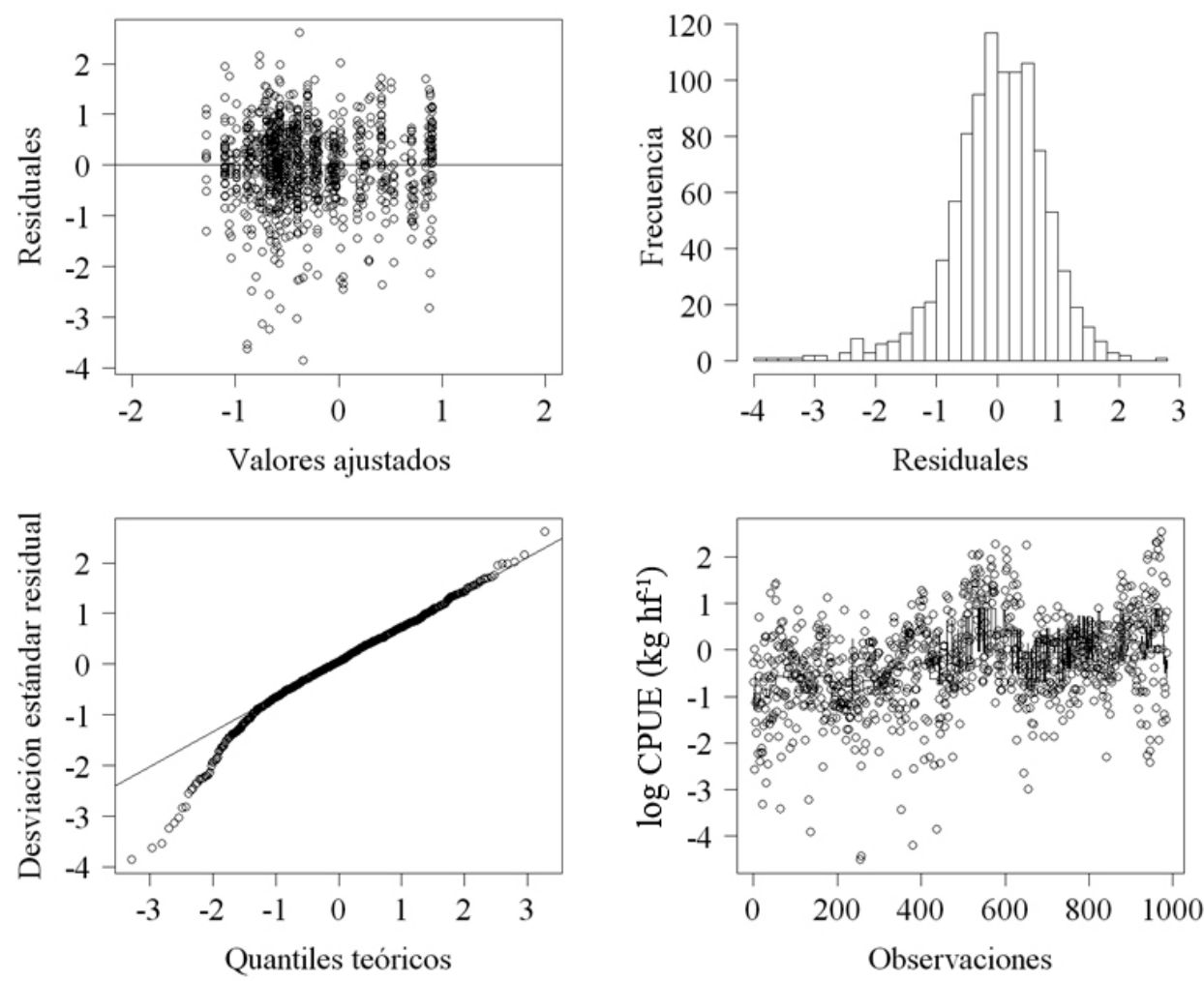

Figura 4

Diagnóstico del modelo lineal generalizado para pargo rayado (Lutjanus synagris)

Diagnostic of the generalized linear model for snapper (Lutjanus synagris) 
del modelo. Los valores estandarizados desde 1994 hasta 1998 mostraron un incremento, alcanzando en este último año un 47\% sobre la media del modelo. Para el año 2001 se observó una reducción en el $\log _{\mathrm{e}}(\mathrm{CPUE})$, situando las estimaciones un 30\% por debajo de la media aunque con un amplio intervalo de confianza (Fig. 5a).

El $\log _{\mathrm{e}}$ (CPUE) estandarizada a nivel intra-anual, mostró los mayores valores a fin de año, superando en los últimos tres meses los valores de la media estimada por el modelo, entre un 25 y 29\%. En mayo los valores estimados estuvieron un $41 \%$ por debajo del valor medio (Fig. 5b). En términos espaciales, en las zonas 1 y 5 correspondientes a Cienaga y Buritaca, se presentaron los mayores valores de $\log _{\mathrm{e}}$ (CPUE), mientras que la zona 2 presentó la menor CPUE asociado a una menor variabilidad (Fig. 5c).

En la escala real $\left(\mathrm{kg} \mathrm{hf}^{-1}\right)$, la CPUE estandarizada mostró un incremento desde 1,1 kg hf ${ }^{-1}$ en 1994 hasta 2,6 kg hf $f^{-1}$ en 1998, para posteriormente disminuir hasta el 2001(1,4 kg hf-1) (Fig. 6a). La CPUE estacionalmente, se incrementa desde mayo $\left(0,82 \mathrm{~kg} \mathrm{hf}^{-1}\right)$ hasta alcanzar los mayores valores entre octubre y diciembre $(1,63 \mathrm{~kg}$ $\mathrm{hf}^{-1}$ en promedio), que continua hasta marzo con 1,39 kg $\mathrm{hf}^{-1}$ (Fig. 6b).
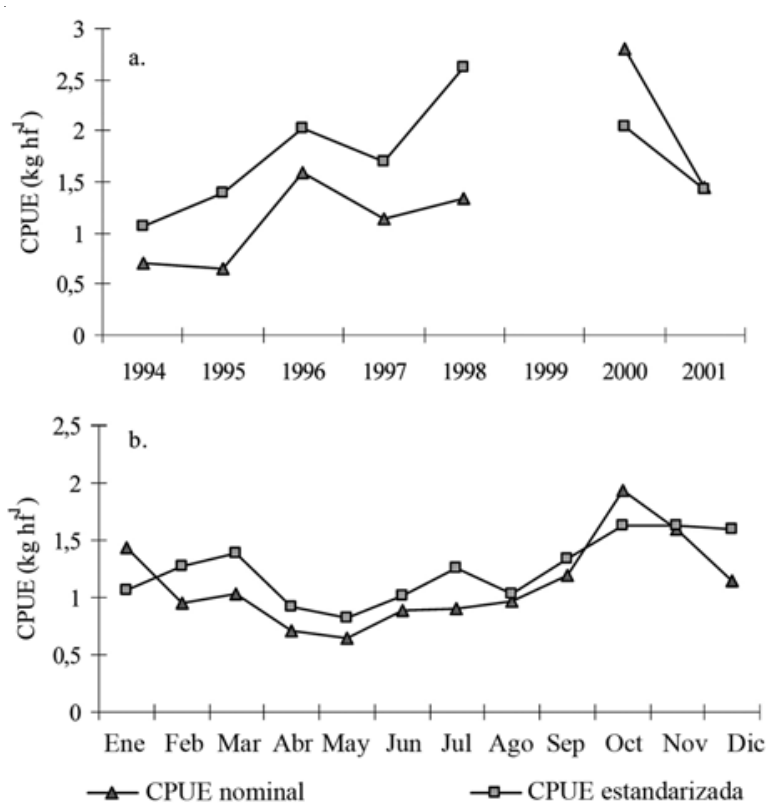

Figura 6

Captura por unidad de esfuerzo nominal y estandarizada en $\mathrm{kg} \mathrm{hf}^{-1}$, a) CPUE a nivel anual b) CPUE a nivel mensual

Catch per unit effort, nominal and standardized in $\mathrm{kg} \mathrm{hf}^{-1}$, a) Annual CPUE, b) Monthly CPUE
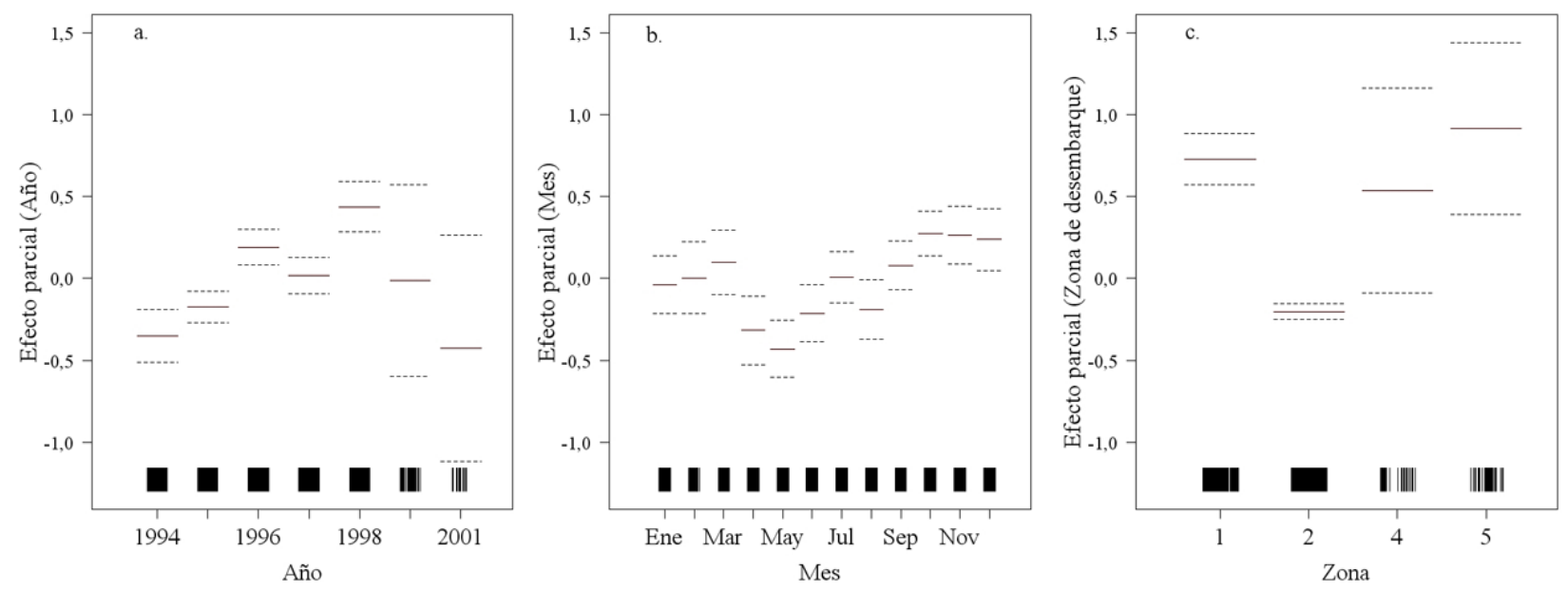

Figura 5

Factores parciales de cada una de las variables incorporadas al modelo lineal generalizado

Partial factors of each one variables included in the generalized linear model 


\section{Correlación entre la variable pesquera y variables oceanográficas}

La descomposición de las series de las variables ambientales proporcionaron los residuos para los análisis de autocorrelación y correlación cruzada. La serie del caudal del río Magdalena mostró durante el periodo analizado una descarga que fluctuó entre 2.533 y 13.375 $\mathrm{m}^{3} \mathrm{~s}^{-1}$. En la escala estacional se evidencian dos máximos dentro del año uno en el mes de julio y otro, con mayor valor, en noviembre. La tendencia de la serie destacó el año 1997 por presentar los menores valores y a 1999 con mayores valores. Sin embargo los residuos continuaron mostrando un ciclo no explicado (Fig. 7a).

La serie de temperatura mostró para el periodo y zona de estudio valores que fluctuaron entre 23,3 y $29,5^{\circ} \mathrm{C}$. A nivel estacional, la temperatura mostró menores valores a principios de año y los más altos en los meses de septiembre y octubre. La serie interanual, mostró los mayores valores a mediados de los años 1995 y 1998 (sobre los $26^{\circ} \mathrm{C}$ ) y valores por debajo de los $25^{\circ} \mathrm{C}$ en los dos últimos años del periodo analizado (Fig. 7b).

La función de autocorrelación de la CPUE mostró una correlación significativa y positiva al primer y segundo mes de retraso $(r=0,45$ y $r=0,21$ respectivamente) y el caudal del río en un retraso al primer mes y después de un año $(r=0,59$ y $r=-0,27$ respectivamente) (Fig. 8). La correlación cruzada mostró correlación negativa y significativa entre la CPUE del pargo rayado y la temperatura superficial con un retraso de 4 meses. Solo se registraron correlaciones significativas y positivas con la descarga del río después de 1, 2, 3 y 11 meses de retraso, consistente con la estacionalidad que persiste en el ruido de esta serie. Entre el caudal del río y la temperatura, se observó una correlación positiva significativa al primer y segundo mes y una correlación significativa e inversa 5 meses antes (Fig. 9).
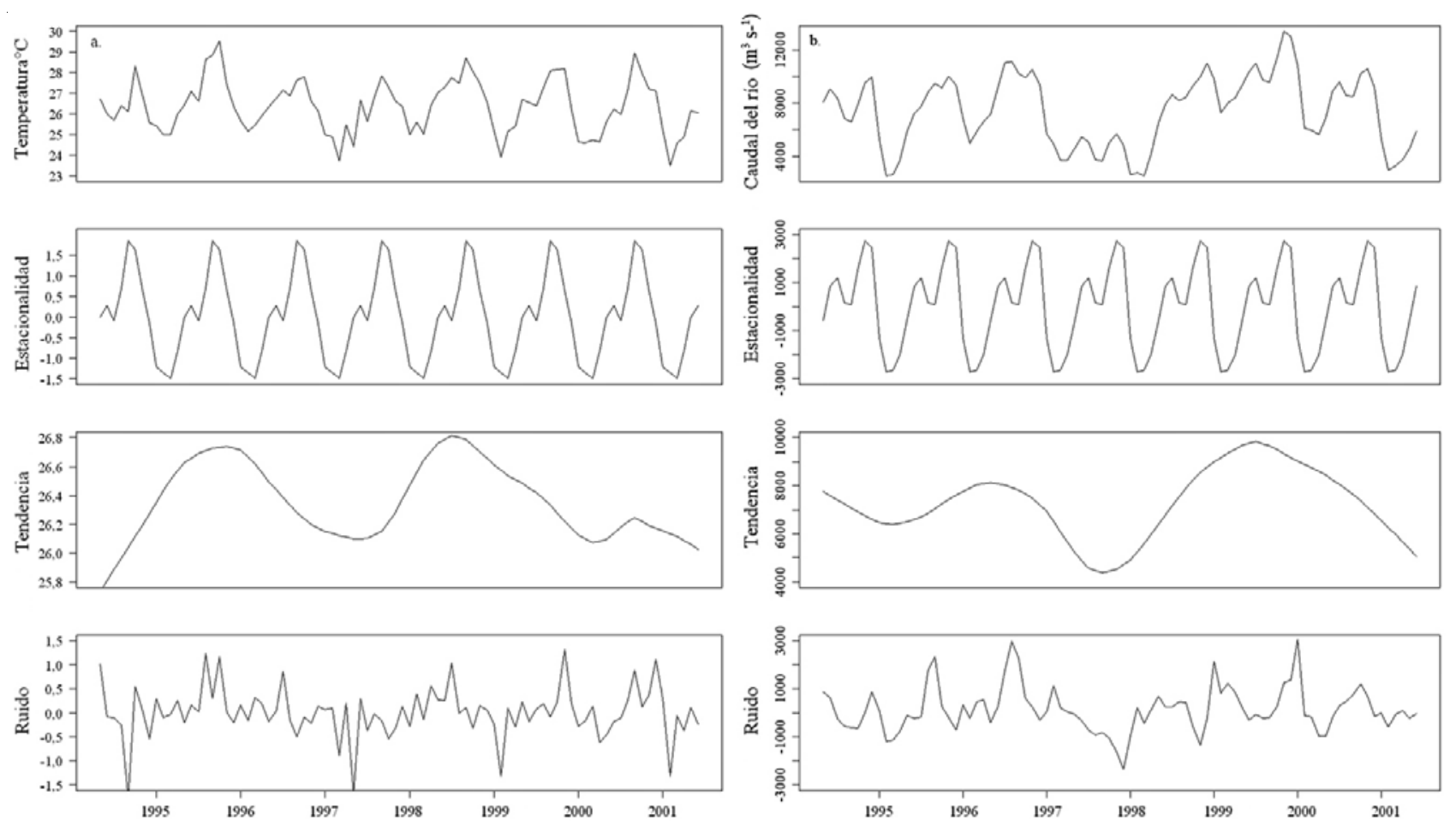

Figura 7 Descomposición de las series de tiempo de las variables ambientales usando un suavizador (STL), periodo mayo de 1994 -
diciembre de 2001. a) Serie de tiempo de la temperatura, b) Serie de tiempo del caudal del río

Time series of environmental variables decomposition using smoothing (STL), from May 1994 to December 2001. a) Time series of temperature, b) Time series of river flow rate 
CPUE $\left(\mathrm{kg} \mathrm{hf}^{1}\right)$

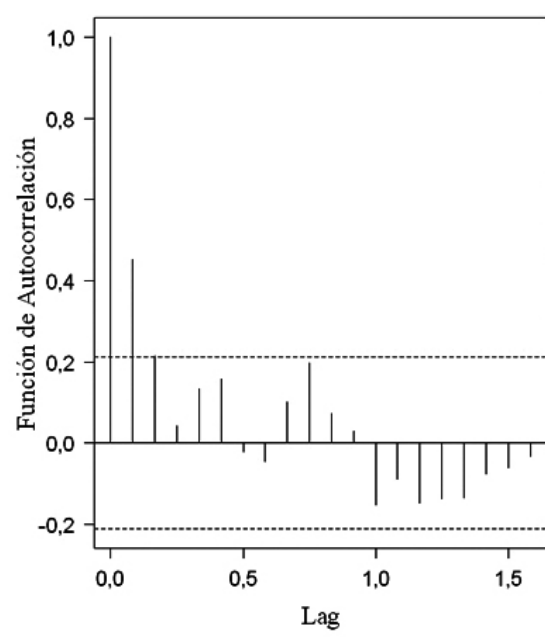

Temperatura ${ }^{\circ} \mathrm{C}$

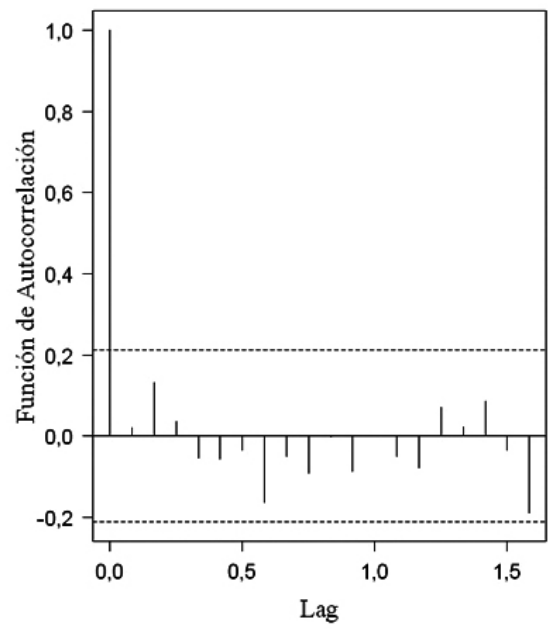

Caudal del río $\left(\mathrm{m}^{3} \mathrm{~s}^{-1}\right)$

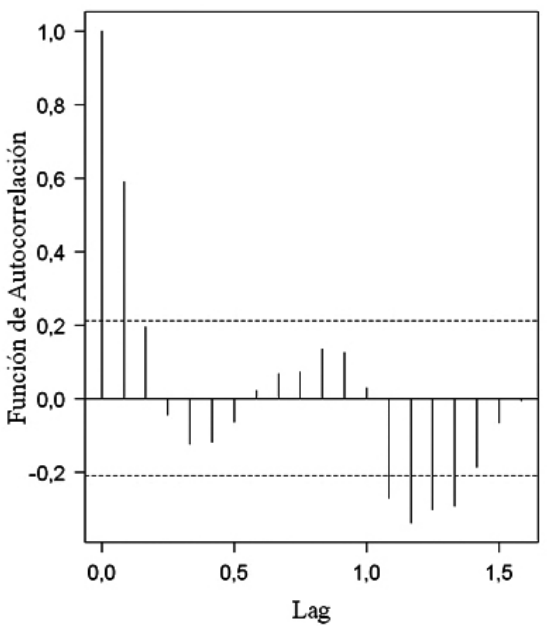

Figura 8

Función de autocorrelación de los residuales de la CPUE de pargo rayado (Lutjanus synagris) y las variables ambientales, la línea puenteada indica el nivel significativo de autocorrelación con $\alpha=0,05$; lag: tiempo de retraso $(1,0=12$ meses)

Autocorrelation function for CPUE of snapper (Lutjanus synagris) residuals and environmental variables, the dotted line show the significant autocorrelation level, $\alpha=0.05$; lag: delay time (1.0 = 12 months)
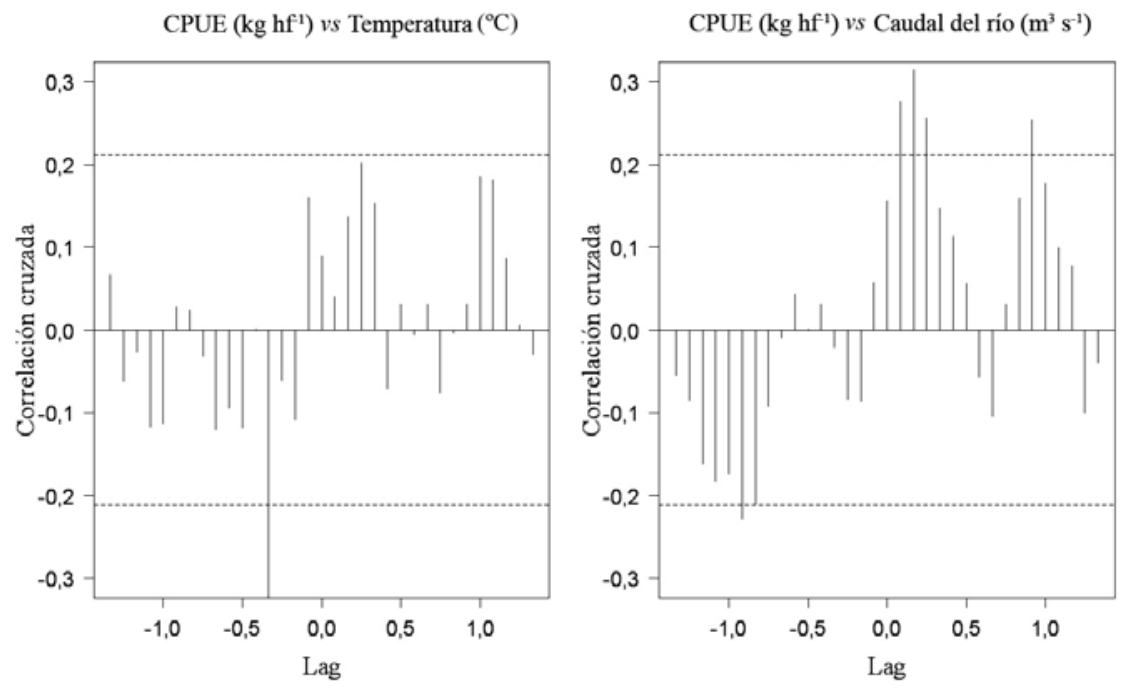

Caudal del río $\left(\mathrm{m}^{3} \mathrm{~s}^{-1}\right)$ vs Temperatura $\left({ }^{\circ} \mathrm{C}\right)$

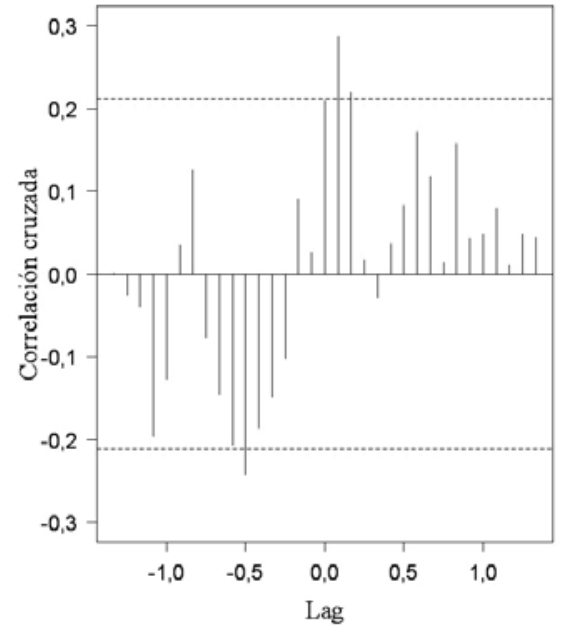

Figura 9

Función de correlación cruzada de los residuales de CPUE de pargo rayado (Lutjanus synagris) y las variables oceanográficas, la línea punteada indica el nivel significativo de correlación con $\alpha=0,05$; lag: tiempo de retraso $(1,0=12$ meses $)$

Cross correlation function for CPUE of snapper (Lutjanus synagris) residuals and oceanographic variables; the dotted line shows the significant correlation level, $\alpha=0.05$; lag: delay time $(1.0=12$ months $)$ 


\section{Discusión}

Uno de los propósitos de este estudio fue detectar fuentes de variabilidad fijas que podrían explicar la captura por unidad de esfuerzo del pargo rayado (Lutjanus synagris) en la PAC del Departamento del Magdalena en el Caribe colombiano. El MLG aplicado a los datos de $\log _{e}(\mathrm{CPUE})$ explicó el 31\% de la variabilidad, siendo el factor año el que representó la mayor devianza explicada. Estos resultados fueron satisfactorios considerando que se utilizó un número bajo de registros individuales (987) durante el periodo de estudio. Los valores anuales mostraron un incremento desde $1,1 \mathrm{~kg} \mathrm{hf}^{-1}$ a partir de 1994 hasta alcanzar un valor máximo en 1998 (2,6 kg $\mathrm{hf}^{-1}$ ). En los últimos años de la serie la CPUE disminuye, con mayor variabilidad y menor número de registros. Aunque no se descarta que los cambios anuales puedan estar relacionados con cambios en la abundancia relativa del pargo rayado en la zona de estudio, los cambios en la CPUE estandarizada podrían estar siendo modulados por el poder de pesca. En efecto, aunque la flota de la PAC no se ha incrementado en número, se ha observado un incremento en el uso de mayor tecnología de pesca. Esto se refiere particularmente a innovación en el material de los cascos e incremento en la motorización, lo que les permite a los pescadores acceder a zonas de pesca más alejadas. A su vez, el incremento en la CPUE desde 1994 a 1998 podría estar relacionado con la disminución en el tamaño de los anzuelos de los palangres (Barros \& Manjarrés 2004), lo que permitiría vulnerar un mayor rango de tamaños y disminuir la selectividad del arte. La tendencia a capturar individuos de menores longitudes con el tiempo, inferiores a la talla óptima de captura (Escobar \& Duarte 2009) podría dar cuenta de esta apreciación.

En la escala estacional, los meses explicaron el 8\% de la variabilidad de la captura por unidad de esfuerzo, mostrando los mayores valores de octubre a diciembre $\left(1,63 \mathrm{~kg} \mathrm{hf}^{-1}\right)$, manteniéndose por encima de la media $\left(1,25 \mathrm{~kg} \mathrm{hf}^{-1}\right)$ hasta marzo. La estacionalidad en la CPUE del pargo rayado podría estar relacionada con la disponibilidad y vulnerabilidad de la especie, condicionada por factores ambientales (Bigelow et al. 1999). La mayor CPUE estimada por el modelo entre octubre y diciembre, estaría relacionada con la época de lluvia (agosto-diciembre) que presenta la zona del Departamento del Magdalena. En efecto, la estación lluviosa se caracteriza por la ausencia de vientos alisios (Giannini et al. 2000) lo que permite una actividad continua de las embarcaciones artesanales permitiendo mayores desembarques (Manjarrés 2004b). Otro factor importante que puede afectar la CPUE estándar en los tres primeros meses del año, se puede atribuir al evento de "bajanza" (Arévalo 1996). Este evento está relacionado con migraciones verticales de individuos de gran tamaño de esta especie y otros pargos, que generalmente ocurren después de fuertes marejadas y favorece la presencia de grandes concentraciones de pargo en la zona costera, aumentado la captura (Manjarrés et al. 2001). Las marejadas contribuyen a aumentar la disponibilidad de alimento en la zona costera. Por su parte, los menores valores de abril y mayo $\left(0,92\right.$ y $0,82 \mathrm{~kg} \mathrm{hf}^{-1}$, respectivamente) estarían relacionados con la época seca cuando se incrementan los vientos alisios, con condiciones desfavorables para el ejercicio de la pesca, traspasándose a la actividad turística (Molinet et al. 1991).

En el área del Magdalena se presentan dos estaciones climáticas marcadas, la estación seca (vientos) de enero a abril, que se caracteriza por la influencia de los vientos alisios del norte que provocan un transporte costa afuera, generando una surgencia de aguas subsuperficiales en la zona costera (Giannini et al. 2000). Esto se ve reflejado en la serie de tiempo de la temperatura superficial del mar, donde la tendencia estacional mostró temperaturas bajas a principios de año, junto con altas concentraciones de clorofila relacionado con el aporte de nutrientes de las aguas surgidas, lo que estimula la mayor producción biológica (Leyva 1998). A su vez, el caudal del río Magdalena disminuye durante esta estación, principalmente por la escasez de lluvias. La estación lluviosa (sin viento), de agosto a diciembre, se caracteriza por la fuertes precipitaciones y mayores valores en el caudal del río Magdalena, alta temperatura y bajas concentraciones de salinidad y clorofila. Estas condiciones estarían determinando variaciones en la CPUE (Beamish 1995, Sinclair \& Frank 1995), ya que en la escala estacional la CPUE se relaciona más con la disponibilidad y capturabilidad que con la abundancia del recurso (Bigelow et al. 1999).

La relación recurso-ambiente es especialmente importante en la zona costera y estuarina, donde la entrada de agua dulce puede afectar la abundancia de las poblaciones de peces (Drinkwater 1986, Quiñonez \& Montes 2001). Algunos autores han encontrado relaciones entre la descarga del río, la abundancia y desembarques o capturas (Cury \& Roy 1989, Runge et al. 1999). De acuerdo al análisis de correlación cruzada, en el presente estudio se detectaron correlaciones entre las variables ambientales analizadas (temperatura superficial del mar y caudal del río Magdalena) y la CPUE del pargo rayado con cierto grado de retraso y particularmente con el caudal del río Magdalena. Aunque muchos estudios que enfrentan la relación entre la abundancia de recursos marinos y las variables ambientales se basan en análisis de correlación, estas 
correlaciones basadas en series de tiempo históricas de variables ambientales y pesqueras han sido bastante criticadas, dado el bajo poder predictivo de los modelos y las posibles correlación espurias (Walters \& Collie 1988). En este trabajo no se encontró una relación significativa entre los residuales de CPUE y la temperatura superficial del mar. No obstante la CPUE del pargo responde positivamente a la influencia del caudal del río Magdalena con uno a tres meses de retraso, lo que podría estar relacionado con un incremento en la productividad e influir positivamente en la disponibilidad de la especie a través de un aumento en la abundancia de presas potenciales, siendo estas principalmente cangrejos y otros crustáceos (Gomez-Chanchong 2004, Hernández et al. 2008). Esto explicaría el conocimiento empírico de los pescadores sobre el fenómeno de la "bajanza del pargo".

En términos espaciales, en el área de operación de la PAC existen alrededor de 20 sitios de desembarque, los que fueron agrupados geográficamente en cinco zonas. En el modelo las zonas 1 y 5 correspondiente a Cienaga Grande de Santa Marta y cabañas de Buritaca, mostraron los mayores valores en las CPUE. La zona de la Cienaga se caracteriza por una alta productividad dado por el aporte del río Magdalena y la zona de Buritaca por ser un litoral rocoso donde se desarrolla una franja estrecha de formaciones coralinas, y pequeñas bahías que presentan complejos ecosistemas, que podrían actuar como zonas de crianza, alimentación y refugio.

A pesar de los bajos niveles de correlación, los resultados de este estudio entregan evidencia que existen cambios en la CPUE del pargo rayado en el Caribe colombiano que son explicados significativamente por factores espacio-temporales. Estos cambios se relacionan con algunas variables ambientales como las analizadas en este trabajo que determinan la productividad de la zona de influencia de la PAC. Por ejemplo, el caudal del río Magdalena y la influencia de los vientos alisios que determinan algunas condiciones de la columna de agua y a su vez el esfuerzo de pesca. Con el objeto de investigar estos y otros factores ambientales sobre la disponibilidad y abundancia del pargo y otros recursos de la PAC, se recomienda continuar desarrollando programas de vigilancia y control de los desembarques e investigación orientada a la obtención de parámetros de historia de vida y de dinámica poblacional.

\section{Agradecimientos}

Este estudio se realizó en el marco del proyecto "Análisis espacial y temporal de la producción pesquera artesanal del norte del mar caribe de Colombia. Insumo para el establecimiento de una pesquería competitiva y responsable" financiado por COLCIENCIAS (Cód. 111734119398), la Universidad del Magdalena-Colombia y la Universidad de Concepción-Chile. Se agradece a Juan Carlos Quiroz del Instituto de Fomento Pesquero de Chile (IFOP) y a Luis Manjarrés de la Universidad del Magdalena por su ayuda en el tratamiento de los datos e información. Se agradece también las facilidades brindadas por Mauricio Braun, Director del Instituto de Fomento Pesquero de Chile (IFOP) en la etapa final de este trabajo, y a la Dirección de Postgrado de la Universidad de Concepción por el co-financiamiento durante la permanencia en el programa de Magíster en Ciencias con mención en Pesquerías del Departamento de Oceanografía.

\section{Literatura citada}

Allen R \& R Punsly. 1984. Catch rates as indices of abundance of yellowfin tuna, Thunnus albacares, in the eastern Pacific Ocean. Inter-American Tropical Tuna Commission Bulletin 18(4): 301-379.

Andrade CA \& E Barton. 2005. The Guajira upwelling system. Continental Shelf Research 25(9): 1003-1022.

Arévalo JC. 1996. Caracterización trófica y reproductiva de las poblaciones de Lutjanus analis (Cuvier, 1828) y Lutjanus synagris (Linnaeus, 1758) en el parque Nacional Natural Tayrona, Caribe colombiano. Tesis de grado. Universidad Jorge Tadeo Lozano, Bogotá, 65 pp.

Barros M \& L Manjarrés. 2004. Inventario y caracterización general de la flota pesquera artesanal del sector norte del Departamento del Magdalena (Taganga-La Jorará) En: Manjarrés L (ed). Pesquerías demersales del área norte del Mar Caribe de Colombia y parámetros biológico pesqueros y poblacionales del recurso pargo, pp. 12-23. Universidad de Magdalena, Santa Marta.

Beamish RJ. 1995. The need to understand the relationship between climate and dynamics of fish populations. En: Beamish RJ (ed). Climate change and northern fish populations, pp. 1-2. Canadian Special Publication of Fisheries and Aquatic Science. National Research Council of Canada, Ottawa.

Bigelow KA, CH Boggs \& X He. 1999. Environmental effects on swordfish and blue shark catch rates in the US North Pacific longline fishery. Fisheries Oceanography 8(3): 178198.

Blanco J. 1998. Las variaciones ambientales estacionales en las aguas costeras y su importancia para la pesca en la región de Santa Marta. Caribe colombino. Tesis M. Sc. Biología Marina, Universidad Nacional de Colombia, Bogotá, 50 pp.

Brockwell PJ \& RA Davis. 2002. Introduction to time series and forecasting, 434 pp. Springer. New York. 
Cleveland RB, WS Cleveland, JE McRae \& I Terpenning. 1990. STL: a seasonal-trend decomposition procedure based on Loess (with discussion), Journal of Official Statistics 6: 3-73.

Correa F \& L Manjarrés. 2004. Inventario y caracterización general de las comunidades económicas de pesca artesanal de la Guajira, Mar Caribe de Colombia En: Manjarrés L (ed). Pesquerías demersales del área norte del Mar Caribe de Colombia y parámetros biológico pesqueros y poblacionales del recurso pargo, pp. 23-35. Universidad del Magdalena, Santa Marta.

Cuello F, LO Duarte, L Manjarrés \& CB García. 2005. Distribución espacial de variables pesqueras y oceanográficas en el área de afloramiento del Mar Caribe de Colombia. Aplicación de un Sistema de Información Geográfica. Universidad del Magdalena, Santa Marta. [CDROM].

Cury P \& C Roy. 1989. Optimal environmental window and pelagic fish recruitment success in upwelling areas. Canadian Journal of Fisheries and Aquatic Science 46: 670-680.

Drinkwater KF. 1986. On the role of freshwater outflow on coastal marine ecosystems- a workshop summary. En: Skreslet S (ed). The role of freshwater outflow in coastal marine ecosystems, pp. 429-438. Springer-Verlag, Berlin.

Duarte LO \& C García. 2002. Testing responses of a tropical shelf ecosystem to fisheries management strategies. A smallscale fishery from the Colombian Caribbean Sea. En: Pitcher T \& K Cochrane (eds). The use of ecosystem models to investigate multispecies management strategies for capture fisheries. Fisheries Centre Research Reports 10(2): 142-149.

Escobar F \& LO Duarte. 2009. Cambios temporales en la estructura de tamaños de Lutjanus synagris (Linnaeus, 1758) capturado en el norte del mar Caribe de Colombia. En: Duarte LO (ed). Análisis espacial y temporal de la producción pesquera artesanal del norte del mar Caribe de Colombia. Insumo para el establecimiento de una pesquería competitiva y responsable, pp. 192-216. Informe Técnico Final, Universidad del Magdalena, Colciencias, Universidad de Concepción, Santa Marta.

García CB, LO Duarte \& D von Schiller. 1998. Demersal fish assemblages of the Gulf of Salamanca, Colombia (southern Caribbean Sea). Marine Ecology Progress Series 174: 13-25.

Giannini A, Y Kushnir \& M Cane. 2000. Interannual variability of Caribbean rainfall, ENSO and the Atlantic Ocean. Journal of Climate 13: 297-311.

Gómez-Canchong P, L Manjarrés, LO Duarte \& J Altamar. 2004. Atlas pesquero del área norte del Mar Caribe de Colombia, 230 pp. Universidad del Magdalena, Santa Marta.

Hernández I, C Aguilar \& G González. 2008. Tramas tróficas de peces de arrecifes en la región noroccidental de Cuba. I.
Contenido estomacal. Revista de Biología Tropical 56(2): 541-555.

Ihaka R \& R Gentleman. 1996. R: A language for data analysis and graphics. Journal of Computational and Graphical Statistics 5: 299-314.

Leyva P. 1998. El medio ambiente en Colombia, 495 pp. Instituto de Hidrología Meteorología y Estudios Ambientales. Ministerio del Medio Ambiente, República de Colombia, Bogotá.

Manjarrés L (ed). 2004a. Estadísticas pesqueras artesanales de los Departamentos del Magdalena y la Guajira, con aplicación en herramientas informáticas para su sistematización y procesamiento, 72 pp. Gente Nueva, Santa Marta.

Manjarrés L (ed). 2004b. Pesquerías demersales del área norte del Mar Caribe de Colombia y parámetros biológicopesqueros y poblacionales del recurso pargo, 318 pp. Ed. Gente Nueva. Universidad del Magdalena, Santa Marta.

Manjarrés L, C García \& A Acero. 2001. Caracterización ecológica de las asociaciones de peces demersales del Caribe colombiano norte, con énfasis en los pargos (Lutjanidae). Boletín de Investigaciones Marinas y Costeras 30: 77-108.

McCullagh P \& JA Nelder. 1989. Generalized Linear Models, 506 pp. St. Edmundsbury Press, Suffolk.

Molinet R, MT Badaracco \& JJ Salaya. 1991. Análisis de series de tiempo aplicado a la pesca del sistema camarónpargo en golfo Triste, Venezuela. Scientia Marina 55(2): 427-437.

Quiñones RA \& RM Montes. 2001. Relationship between freshwater input to the coastal zone and the historical landings of the benthic/demersal fish Eleginops maclovinus in central-south Chile. Fisheries Oceanography 10(4): 311328.

Runge JA, M Castonguay, Y de Lafontaine, M Ringuette \& JL Beaulieu. 1999. Covariation in climate, zooplankton biomass, and mackerel recruitment in the southern Gulf of St. Lawrence. Fisheries Oceanography 8(2): 139-149.

Sinclair M \& KT Frank. 1995. Symposium summary. En: Beamish J (ed). Climate change and northern fish populations, pp. 735-739. Canadian Special Publication of Fisheries and Aquatic Science. National Research Council of Canada, Ottawa.

Venables WN \& BD Ripley. 2002. Modern applied statistics with S, 481 pp. Springer, New York.

Walters CJ \& JS Collie. 1988. Is research on environmental factors useful to fisheries management? Canadian Journal of Fisheries and Aquatic Science 45: 1848-1854.

Zuur A, E Ieno \& G Smith. 2007. Analysing ecological data, 667 pp. Springer, New York. 